\title{
El léxico de las patologías hipiátricas en Instruçam da Cavallaria de Brida de Antonio Pereyra Rego frente al léxico de sus fuentes
}

The lexicon of horse pathologies in Instruçam da Cavallaria de Brida by Antonio Pereyra Rego confronted with the lexicon of its sources

\author{
ALEXANDRA SOARES RODRIGUES \\ ESE-Instituto Politécnico de Bragança \\ CELGA-ILTEC-Universidade de Coimbra \\ afsr@ipb.pt
}

Resumen: El objetivo de este trabajo consiste en comparar el léxico de las patologías hipiátricas presentes en el manual de albeitería de Antonio Pereyra Rego, de 1679, Instruçam da Cavallaria de Brida: tratado unico, dedicada ao invicto martyr S. Jorge con el léxico presentado en sus fuentes. El autor menciona a muchos de los tratados extranjeros en que se ha basado para redactar este trabajo. La comparación entre el léxico de las patologías de los caballos en portugués, en francés, en italiano y en castellano emergente en las diferentes fuentes apuntadas por Rego nos permite averiguar si la influencia de esos tratados sobre Rego solamente se observa en el dominio conceptual o bien si también se produjo en el dominio terminológico.

Palabras clave: albeitería; léxico portugués de la ciencia y de la técnica; patologías hipiátricas.

Abstract: The aim of this work consists in the comparison between the lexicon of horse pathologies which is present in the treatise of veterinary medicine, Instruçam da Cavallaria de Brida: tratado unico, dedicada ao invicto martyr S. Jorge, by Antonio Pereyra Rego, published in 1679, and the lexicon which is present in the sources of this book. The author mentions many foreign treatises in which he had based the redaction of this work. The comparison between the lexicon of horse diseases in Portuguese, in French and in Spanish, which emerges in the several sources that are mentioned by Rego, allows us to investigate if the influence of those treatises on Rego is only observable in a conceptual domain or if it also produced results in the terminological field.

Key words: Veterinary medicine; Portuguese lexicon of science and technics; horse pathologies.

Fecha de presentación: 21/11/2018 Fecha de aceptación: 05/05/2019.

\section{INTRODUCCIÓN}

El presente trabajo pretende identificar las correspondencias terminológicas entre el tratado Instruçam da Cavallaria de Brida, de Antonio Pereyra Rego, publicado en 1679, escrito en portugués, y sus fuentes en castellano, francés e italiano, focalizando nuestra atención en la posibilidad de haber una correspondencia etimológica entre los términos en las cuatro lenguas. En Rodrigues (2013), hemos procedido a una introducción de este

${ }^{*}$ Este trabajo se integra en el proyecto Léxico Portugués de la Ciencia y de la Técnica (LEXPORTEC).

ALEXANDRA SOARES RODRIGUES,

«El léxico de las patologías hipiátricas en Instruçam da Cavallaria de Brida de Antonio Pereyra Rego frente al léxico de sus fuentes», Revista de Lexicografia, XXV (2019), pp. 253-270 ISSN: 1134-4539, e-ISSN: 2603-6673. DOI: https://doi.org/10.17979/rlex.2019.25.0.6004 
tema, planteando los problemas que surgen de un análisis comparativo de este tipo. En el presente trabajo, desarrollaremos los resultados obtenidos a través de esa comparación. Este trabajo se sitúa en un dominio más amplio de análisis del léxico portugués de la albeitería que estamos llevando a cabo y que va demostrando sus frutos en Rodrigues (2012a, 2012b, 2013, 2016, 2017a, 2017b) y Rodrigues y Sá Morais (2015).

Rego (1679: 185) apunta numerosos tratados de diferentes lenguas como sus fuentes, desde los clásicos de la Antigüedad (Publio Vegecio), pasando por los clásicos medievales (Giordano Ruffo), hasta los compendios sus contemporáneos de grande divulgación (Ruini). Es imposible determinar si Rego efectivamente consultó a todos esos tratados, especialmente los más antiguos, o bien si los nombró por una cuestión de atestación de autoridad. Por estas razones, nos parece más plausible que Rego haya consultado a los manuales contemporáneos. Teniendo en cuenta esta hipótesis y siguiendo los criterios de que los tratados apuntados como fuentes fueran:

a) escritos en lenguas distintas;

b) contemporáneos de Rego;

c) de gran divulgación y prestigio científico en la época.

Para nuestro trabajo, hemos escogido las siguientes fuentes: el francés Jacques de Solleysel, Le Parfait mareschal qui enseigne à connoître la beauté, la bonté et les défauts des chevaux, les signes et les causes des maladies... (1654); el boloñés Carlo Ruini, Anatomia del cavallo, infermita et suoi remedii (1618); el español Miguel de Paracuellos, Libro de albeyteria (1658).

\subsection{Descripción de las obras analizadas}

Haremos en seguida una breve descripción de cada una de las obras analizadas.

1.1.1. Instruçam da Cavallaria de Brida: tratado unico, dedicada ao invicto martyr $S$. Jorge, por Antonio Pereyra Rego, 1679

Este tratado tiene sucesivas reediciones en 1693, 1712, 1717 y 1731. El tratado está dividido en dos partes, como la mayoría de los tratados de albeitería, no solamente de la época, sino también de épocas pretéritas (e.g. Livro de Alveitaria de Mestre Giraldo de 1318). La primera parte trata de la equitación. La segunda parte, que es objeto de nuestro enfoque, trata de la albeitería. La organización de los temas en la parte de la albeitería es la siguiente: patologías generales y modos de emplear tratamientos, patologías de la cabeza, del corazón, de las patas delanteras, heridas, patologías respiratorias, fiebres, patologías en el sistema digestivo, riñones y patas posteriores. Hay capítulos entre los cuales no existe una verdadera continuidad. Eso se debe al hecho de que, por ejemplo, no se traten los miembros posteriores seguidamente a los miembros anteriores. Para cada patología, el autor identifica los respectivos síntomas, las medicinas, el modo de preparación y la posología de las medicinas y otras informaciones necesarias a que la enfermedad sea vencida. Contiene la ilustración de un caballo con indicación de las partes anatómicas y sus patologías. 


\subsubsection{Jacques de Solleysel, Le parfait Mareschal, 1654}

El tratado de Solleysel tiene una primera edición en 1654 y una última en 1782. El tratado fue tan divulgado que tuvo 30 ediciones en Francia. Le Parfait Mareschal contiene las dos clásicas partes dedicadas a la albeitería (la primera) y a la equitación (la segunda). La parte dedicada a la albeitería muestra que Rego se podría haber apoyado fuertemente en este manual para su tratado, una vez que la organización de los contenidos en Rego y en Solleysel es muy similar. Así, Solleysel empieza con las patologías de la cabeza, sigue con las patologías de carácter general que afectan la cabeza, patologías del sistema digestivo, patologías neurológicas, patologías de los miembros de locomoción. En seguida, se dedica a patologías que afectan a todo el organismo, patologías respiratorias, del corazón, la fiebre, miembros traseros, entre otros. Solleysel describe las medicinas muy detalladamente y apunta diferentes medicinas para la misma patología. Sin embargo, las patologías están escasamente descritas. Este tipo de planteamiento hace presuponer que este manual es adecuado a un público que conozca profundamente las patologías y que busque sobre todo el modo de curarlas. Contiene ilustraciones.

\subsubsection{Carlo Ruini, Anatomia del cavallo, infermita et suoi remedii, 1618}

La edición que hemos consultado de este tratado es de 1618. Pero su primera edición es de 1598. Este es el más completo, sistemático y profundo manual de albeitería en Europa de la época que estamos analizando. Por su carácter riguroso, detallado y sistemático, el manual de Ruini es adecuado a los que empiezan a estudiar este dominio científico. Es un tratado específico de medicina, por lo que no contiene ninguna parte dedicada a la equitación. Está constituido por dos partes. La primera parte está dedicada a la descripción muy pormenorizada de la anatomía de los caballos y contiene ilustraciones detalladas. La segunda parte versa sobre las patologías. Para cada patología se enseña la definición y la identificación de la patología, los síntomas, las causas, las medicinas, el modo de preparación de las medicinas y su posología y otros modos de actuar para que la cura sea conseguida. La organización de las materias en la segunda parte tiene en cuenta los sistemas afectados (p.ej. patologías del sistema reproductor, patologías del sistema digestivo, del neurológico), los miembros afectados (cabeza, ojos, miembros de locomoción) y el tipo de patología (tumores, roturas, relajaciones, entre otros).

\subsubsection{Miguel de Paracuellos, Libro de albeyteria, 1658}

La primera edición de Paracuellos es de 1629. Hemos consultado la edición de 1658. La edición de 1702 presenta un añadido del arte de herrar de 1702.

De los cuatro manuales analizados, el de Paracuellos es el menos sistemático. Está constituido por una sola parte, que versa sobre la albeitería. La organización de las materias es la siguiente: patologías digestivas, patologías oculares, patologías neurológicas, patologías generales, patologías de los miembros de locomoción, seguidas de sus heridas, nuevamente patologías generales, nuevamente patologías de las patas. Hay ocasiones en que se presenta un inventario de patologías, pero sin que se describan. No contiene ilustraciones. 
Nuestro análisis ha consistido en establecer las correspondencias entre las patologías tratadas en los cuatro manuales. En la sección 4, se explicitan por medio de tablas esas correspondencias. Por limitaciones de espacio, solamente nos detendremos en enfatizar las correspondencias etimológicas halladas. El lector puede observar las restantes correspondencias entre patologías en las tablas.

\section{DIFICULTADES Y FACILITACIONES EN EL ANÁLISIS COMPARATIVO}

La comparación del léxico en Rego, Ruini, Solleysel y Paracuellos está dificultada por discrepancias múltiples que hemos descrito detenidamente en Rodrigues (2013) y que presentemente se pueden sintetizar en:

i) diferentes grados de profundidad y sistematicidad de cada autor frente a una patología;

ii) la categorización poco sistemática de las patologías en Rego, Solleysel y Paracuellos, que contrasta con la categorización muy cuidada de Ruini;

iii) la taxonomía de las patologías. En Ruini se describen patologías y sus subtipos, mientras que en los restantes autores las patologías encierran categorías más generales;

iv) el hecho de que una serie de síntomas corresponda en un autor a una sola patología y en otro autor cada síntoma corresponda a diferentes patologías;

v) la atribución por los autores de causas distintas a un mismo síntoma o patología;

vi) la correspondencia entre el significante de los términos, pero no entre los significados;

vii) la insuficiencia de la caracterización de la patología;

viii) la utilización por un autor de un solo término para dos patologías que en otros autores son designadas por términos distintos;

ix) las diferentes conceptualizaciones de la anatomía.

Aunque las dificultades de este tipo de trabajo sean muchas, hay también en los tratados indicios que facilitan la comparación terminológica. Son las siguientes las situaciones que vuelven la comparación posible:

i) indicación en Rego de los términos correspondientes en las otras lenguas;

ii) informaciones metalingüísticas que explicitan la motivación de la terminología, por ejemplo, explicitación de metáforas que están en la base del término.

\section{CATEGORÍAS DE PATOLOGÍAS}

Una vez que hay discrepancia entre la sistematización y organización de las patologías en los cuatro autores estudiados, hemos procedido a una categorización de las enfermedades que en seguida explicitamos:

i) patologías generales, que afectan todo el organismo (e.g. raiva, febre);

ii) heridas debidas a causas externas (e.g. encabrestaduras, chagas \& feridas venenosas, feitas por animaes raivosos, \& danados animais);

iii) patologías neurológicas (e.g. espasmo, vertigio);

iv) patologías de la cabeza y del cuello (e.g. erisipela, mal da fava); 
v) patologías del sistema digestivo y bazo (e.g. torcilhões, fluxo de ventre);

vi) patologías del sistema reproductor (e.g. cascar della matrice, inchação dos testículos);

vii) patologías de las articulaciones y esqueleto (e.g. esforço ou rendimento das pás, cavalo aberto);

viii) patologías de los miembros de locomoción, como grietas o semejantes (e.g. formigueyro, quartos);

ix) patologías de los miembros de locomoción, como tumores (e.g. alifafes, agrião);

x) patologías cardíacas (e.g. latejar do coração, ante cor);

xi) patologías oftálmicas (e.g. cavalo lunático, fluxão dos olhos);

xii) patologías del sistema respiratorio (e.g. polmoeira, tosse).

\section{CORRESPONDENCIAS ENTRE LOS TÉRMINOS}

En esta sección, se explicitan las correspondencias entre las patologías en los cuatro manuales. Recordamos que, por limitaciones de espacio, solamente haremos referencia en el texto a las correspondencias etimológicas entre los términos. El lector podrá identificar en las tablas las correspondencias entre las designaciones de las patologías que no presentan el mismo término etimológicamente relacionado.

\subsection{Patologías generales, que afectan todo el organismo}

Con respecto a las patologías generales, o sea, que afectan todo el organismo (Tabla 1), existe equivalencia etimológica entre Rego y Paracuellos con respecto a las patologías designadas por el autor portugués como agoamento y sarna. Los términos febre y raiva presentan correspondencia etimológica con los términos en francés y en italiano. El término mormo presenta correspondencia con el castellano muermo y con el francés gourme. El término farcin se corresponde a la designación en francés, mientras en la misma enfermedad el término verme tiene correspondencia con la designación utilizada por Ruini.

\begin{tabular}{|c|c|c|c|}
\hline REGO & PARACUELLOS & RUINI & SOLLEYSEL \\
\hline $\begin{array}{l}\text { Agoamento } \\
=\text { resfriamento= } \\
\text { Infusura } 376-377\end{array}$ & $\begin{array}{l}\text { Aguadura } 124 \neq \\
\text { Resfriadura } 119\end{array}$ & & Fourbure 422 \\
\hline Birra 238 & & & Ticq 11 \\
\hline $\begin{array}{l}\text { Febre } 352-360 \\
\text { Febre simples p. } 355 \\
\text { Febres podres p. } 358 \\
\text { Febre pestilencial p. } 360\end{array}$ & & $\begin{array}{l}\text { Febbre terzana/quartana } \\
19 / 20\end{array}$ & $\begin{array}{l}\text { Fiévre } 371 \\
\text { Simples } \\
\text { Putride \& humoralle } \\
\text { Pestilentielle } 372 \\
\end{array}$ \\
\hline Raiva 339 & & Rabbia \& furore 41 & Rage 309 \\
\hline $\begin{array}{l}\text { cavallo magro, \& es- } \\
\text { taçado, que não quer } \\
\text { tornar a medrar } 350\end{array}$ & & & $\begin{array}{l}\text { Chevaux alterez de } \\
\text { flanc } 320 \text { maigres } \\
352 \\
355 \\
\text { Chevaux fortraits } \\
361\end{array}$ \\
\hline$?$ & & $\begin{array}{l}\text { Carboncelli } 24=\text { male del } \\
\text { Mazzo=pestilenza } 25\end{array}$ & \\
\hline
\end{tabular}




\begin{tabular}{|c|c|c|c|}
\hline $\begin{array}{l}\text { Mormo } 254 \text { 'tumor } \\
\text { entre as queixadas' }\end{array}$ & & & Gourme 62 \\
\hline \multirow[t]{4}{*}{$\begin{array}{l}\text { Mormo } 257 \text { 'doença } \\
\text { com espirros e fluxo } \\
\text { pelo nariz' }\end{array}$} & Muermo 83 & $\begin{array}{l}\text { ¿??freddura (ò coriza) é } \\
\text { doença leve } 65\end{array}$ & $\begin{array}{l}\text { Chevaux qui jettent } \\
\text { leur gourme impar- } \\
\text { faitement par les } \\
\text { nazeaux } 33\end{array}$ \\
\hline & & & Fausse gourme 36 \\
\hline & Zimorra 75 & ?Cimoro 67 & Morve 48-49 \\
\hline & & ¿?Catarro ou rheuma 68-69 & $\begin{array}{l}\text { Rhume ou morfon- } \\
\text { dement } 39\end{array}$ \\
\hline sarna 403 & Sarna & Rogna/scabbia29 & Galle 280,434 \\
\hline $\begin{array}{l}\text { Mal de Olanda } 362 \text { ver } \\
\text { tipos } \\
\text { Explicita farcin e ver- } \\
\text { mes }\end{array}$ & & $\begin{array}{l}\text { Mal del verme 26, } 31 \\
\text { (incuído em Contagione 25) } \\
\text { Ver tipos }\end{array}$ & Farcin 359 tipos 363 \\
\hline $\begin{array}{l}\text { ebuliçoens do sangue } \\
374\end{array}$ & & & $\begin{array}{l}\text { Ebulitions du sang } \\
411\end{array}$ \\
\hline \multirow[t]{3}{*}{$\begin{array}{l}\text { graixa, ou gordura } \\
\text { derretida } 375\end{array}$} & & & Gras-fondure 415 \\
\hline & & & Gangrenne 287 \\
\hline & & & $\begin{array}{l}\text { Playes sur le boulet, } \\
\& \text { sur les parties } \\
\text { nerveuses } 306\end{array}$ \\
\hline 6 & & Lepra 27 & \\
\hline$?$ & & Marasmo 152-153 & \\
\hline
\end{tabular}

Tabla 1. Patologías generales que afectan todo el organismo

\subsection{Heridas debidas a causas externas}

En las heridas provocadas por causas externas (Tabla 2), hay correspondencias etimológicas entre el término encabrestadura y los correspondientes en castellano y en francés.

\begin{tabular}{|l|l|l|l|}
\hline REGO & PARACUELLOS & RUNI & SOLLEYSEL \\
\hline $\begin{array}{l}\text { Das chagas dos rins, ou } \\
\text { feridas penetrantes do } \\
\text { corpo do cavallo 337 }\end{array}$ & & $\begin{array}{l}\text { Playes sur le } \\
\text { roignon ¿?? Tb } \\
\text { pode ser testícu- } \\
\text { lo301 }\end{array}$ \\
\hline Encabrestaduras 300 & Encabestraduras & & $\begin{array}{l}\text { Enchevestrures } \\
501,506\end{array}$ \\
\hline $\begin{array}{l}\text { Alcançadura?? } \\
303\end{array}$ & $\begin{array}{l}\text { Rozadura de } \\
\text { herradura }\end{array}$ & & \\
\hline Alcançadura?? & cazapatazo & & $\begin{array}{l}\text { Morsures de beste } \\
\text { veneneuse 313 }\end{array}$ \\
\hline $\begin{array}{l}\text { Das chagas, \& feridas } \\
\text { venenosas, feitas por } \\
\text { animaes raivosos, \& } \\
\text { danados animais p. 339 }\end{array}$ & & & \\
\hline
\end{tabular}

Tabla 2. Heridas debidas a causas externas 


\subsection{Patologías neurológicas}

En las patologías neurológicas, son correspondientes etimológicos en portugués, italiano y francés los términos para vertigio. El término portugués espasmo presenta correspondencia etimológica en castellano e italiano.

\begin{tabular}{|c|c|c|c|}
\hline REGO & PARACUELLOS & RUINI & SOLLEYSEL \\
\hline & & Frenesia 39 & \\
\hline & & Pazzia 45 & \\
\hline & & Capostorno 45 & \\
\hline & & Capogatto 47 & \\
\hline \multirow[t]{5}{*}{ Vertigio 269} & & Vertigine 47 & Vertige 130 \\
\hline & & $\begin{array}{l}\text { Mal caduco (detto da Greci } \\
\text { Epilepsia) } 48\end{array}$ & \\
\hline & & $\begin{array}{l}\text { Apoplesia (volgarmente la } \\
\text { goccia) } 51\end{array}$ & \\
\hline & & Lethargìa 52 & \\
\hline & & Doglia del capo 62 & \\
\hline Desvario 270 & & - & \\
\hline Espasmo $266 ?$ & ??Pasmo 63 & $\begin{array}{l}\text { Spasimo } 57 \\
\text { (general y particular) }\end{array}$ & $\begin{array}{l}\text { Mal du cerf?? } \\
96\end{array}$ \\
\hline $\begin{array}{l}\text { Espasmo (general o } \\
\text { particular) ???266 }\end{array}$ & $\begin{array}{l}\text { Perlesía } 71 \\
\text { (general o de parte } \\
\text { del cuerpo) }\end{array}$ & $\begin{array}{l}\text { Paralisia (ò resolutione de i } \\
\text { neruì) (sólo de parte del } \\
\text { cuerpo) } 54\end{array}$ & \\
\hline
\end{tabular}

Tabla 3. Patologías neurológicas

\subsection{Patologías de la cabeza y del cuello}

Con respecto a las patologías de la cabeza y del cuello, se corresponden etimológicamente en portugués, italiano y francés las designaciones para fava y barbeloens. Entre el portugués y el francés, se corresponden etimológicamente las patologías de sobredentes o dente de lobo y de olivas.

\begin{tabular}{|l|l|l|l|}
\hline REGO & PARACUELLOS & RUINI & SOLLEYSEL \\
\hline Fava 239 & & $\begin{array}{l}\text { Lampasco= male della fava } \\
112\end{array}$ & Lampas ou féve \\
\hline- & & $\begin{array}{l}\text { Risolutione della lingua, ò } \\
\text { paralisia della lingua 108 }\end{array}$ & \\
\hline- & $\begin{array}{l}\text { Lingua macchiata di bianco } \\
108\end{array}$ & \\
\hline Lingua ferida 242 & & Enfiagione della lingua 109 & \\
\hline & & Mal del Pinzanese 109 & \\
\hline Lingua ferida 242 & & $\begin{array}{l}\text { Rottura ò tagli della lingua } \\
109\end{array}$ & \\
\hline çapinhos ou barbeloens & & Barbule 111 & $\begin{array}{l}\text { Barbes ou barbi- } \\
\text { 240 }\end{array}$ \\
\hline & & $\begin{array}{l}\text { Aphte \& vlceri serpiginosi } 11 \\
111\end{array}$ & \\
\hline
\end{tabular}




\begin{tabular}{|c|c|c|}
\hline boca chea 239 & Palatina 112 & \\
\hline $\begin{array}{l}\text { Sobredentes ou dentes } \\
\text { de lobo } 240\end{array}$ & $\begin{array}{l}\text { Denti che nascono fuori } \\
\text { dell'ordine de gl'altri } 113\end{array}$ & $\begin{array}{l}\text { Sur-dents, ou } \\
\text { dentes de loup } 12\end{array}$ \\
\hline Boca ferida 241 & & $\begin{array}{l}\text { Bouche blessée ou } \\
\text { entamée } 13\end{array}$ \\
\hline \multirow[t]{2}{*}{ Fastio 243} & & $\begin{array}{l}\text { Cheval dégoûté } \\
15\end{array}$ \\
\hline & Posteme nelle gengiue 114 & \\
\hline ¿mormo? Landoas??? & Bottoli 114 & \\
\hline ¿mormo? Landoas??? & Floncella 114 & \\
\hline- & Morsea 114 & \\
\hline \multirow[t]{2}{*}{$? ? ?$} & Schirantia 115 & \\
\hline & Strangoglione 119 & \\
\hline $\begin{array}{l}\text { Olivas } 271 \text { [refiere } \\
\text { avives en su texto] }\end{array}$ & Viuole 122 & Avives 99 \\
\hline \multirow[t]{2}{*}{ Erisipela 264} & & $\begin{array}{l}\text { Mal de tête, } \\
\text { nommé mal de } \\
\text { feu } 67\end{array}$ \\
\hline & & $\begin{array}{l}\text { Mal de teste, } \\
\text { qu'on nomme mal } \\
\text { d'Espagne } 69 \\
\end{array}$ \\
\hline \multirow[t]{2}{*}{ Alvarazos 270-271 } & Scroffole 125 & \\
\hline & $\begin{array}{l}\text { Dolore delle orecchie \& } \\
\text { posteme interiori } 101\end{array}$ & \\
\hline \multirow{4}{*}{$\begin{array}{l}\text { Chagas ou ulceras de } \\
\text { dentro das ventas } 249\end{array}$} & Ozene 106 & \\
\hline & Polipi 107 & \\
\hline & $\begin{array}{l}\text { Flusso del sangue del naso } \\
105\end{array}$ & \\
\hline & Sordezza 99 & \\
\hline $\begin{array}{l}\text { fluxo de sangue pella } \\
\text { boca } 247\end{array}$ & $\begin{array}{l}\text { Sangue ch'esce per bocca } \\
151\end{array}$ & Emorragie 93 \\
\hline
\end{tabular}

Tabla 4. Patologías de la cabeza y del cuello

\subsection{Patologías del sistema digestivo y bazo}

En las patologías del sistema digestivo y del bazo, hay paralelismo etimológico entre el portugués y el castellano con respecto a torcilhões y cameras. Entre el portugués y el italiano, las correspondencias etimológicas existen con respecto a lombrigas.

\begin{tabular}{|l|l|l|l|}
\hline REGO & PARACUELLOS & RUINI & SOLLEYSEL \\
\hline Torcilhões 82???? & Torzones 21???? & $\begin{array}{l}\text { Dolor dello stomacho 157- } \\
158\end{array}$ & \\
\hline- & & $\begin{array}{l}\text { Bulimo, ò canina appetenza } \\
160\end{array}$ & Faim-vale 508 \\
& & $\begin{array}{l}\text { Dolor del corpo 161-162 } \\
\text { 6 espécies }\end{array}$ & $\begin{array}{l}\text { Tranchée } 102 \\
\text { 6 especies }\end{array}$ \\
\hline Torcilhões 382 & Torzones 21 & $\begin{array}{l}\text { Flusso del corpo 165 } \\
\text { diarée 460 }\end{array}$ \\
\hline camespécies & 10 espécies & espécies: \\
ventre p. 395 & Camaras130 & Lienteria & \\
& & & \\
\hline
\end{tabular}




\begin{tabular}{|c|c|c|}
\hline & $\begin{array}{l}\text { Ragiatura } \\
\text { Diarrea } \\
\text { disenteria }\end{array}$ & \\
\hline \multirow[t]{3}{*}{ - } & Iliaca 171 [tb é fluxo] & \\
\hline & Colica 174 & \\
\hline & $\begin{array}{l}\text { Dolore humorale, che viene } \\
\text { frà il peritoneo, \& gli intesti- } \\
\text { ni } 176\end{array}$ & \\
\hline \multirow[t]{4}{*}{ Lombrigas 399} & $\begin{array}{l}\text { Vermi, ò lombrici, ò tignole } \\
177\end{array}$ & Vers 441 \\
\hline & $\begin{array}{l}\text { Vscita, \& cascata } \\
\text { dell'intestino retto } 180\end{array}$ & $\begin{array}{l}\text { Des chevaux } \\
\text { ausquels le fon- } \\
\text { dement sort } 466\end{array}$ \\
\hline & Oppilatione del fegato 183 & \\
\hline & Itteritia 185 & \\
\hline \multirow[t]{3}{*}{$\begin{array}{l}\text { Achaques, \& enfremi- } \\
\text { dades da cabeça } 261\end{array}$} & & $\begin{array}{l}\text { Maux de teste } \\
\text { causez d'humeurs } \\
\text { bilieuses } 64\end{array}$ \\
\hline & $\begin{array}{l}\text { Hidropisia } 187 \\
\text { Iposarca, ò anafarca, ò } \\
\text { flemma bianco, ò leu- } \\
\text { coflegmatia } \\
\text { Ascite } \\
\text { Timpanite, ò timpania }\end{array}$ & \\
\hline & $\begin{array}{l}\text { Enfiagione, \& durezza della } \\
\text { milza } 190\end{array}$ & \\
\hline
\end{tabular}

Tabla 5. Patología del sistema digestivo y bazo

\subsection{Patologías del sistema reproductor}

En las patologías del sistema reproductor, hay correspondencias etimológicas entre el portugués y el francés con respecto a la inchação dos testiculos.

\begin{tabular}{|l|l|l|l|}
\hline REGO & PARACUELLOS & RUINI & SOLLEYSEL \\
\hline & & & $\begin{array}{l}\text { Du cheval qui } \\
\text { pisse le sang 124 }\end{array}$ \\
\hline $\begin{array}{l}\text { Inchação dos testiculos } \\
409 \text { parece incluir hernia }\end{array}$ & & & $\begin{array}{l}\text { Fourreau \& les } \\
\text { testicules enflez } \\
156 \\
\text { Enflure de testi- } \\
\text { cules 455 }\end{array}$ \\
\hline & & & $\begin{array}{l}\text { Testicules meur- } \\
\text { tris, enflez ou } \\
\text { endurcis 458 }\end{array}$ \\
\hline & & & \\
\hline & & & \\
& & Posteme, \& vlceri de i testi- & \\
\hline & & Heli 195 & \\
\hline & & Priapismo, \& satiriasi 198 & \\
\hline & & Uscita del membro 199 & \\
\hline
\end{tabular}




\begin{tabular}{|l|l|l|l|}
\hline & & $\begin{array}{l}\text { Sfilato, che da se getta il } \\
\text { seme 200 }\end{array}$ & \\
\hline & & Tarli delle verga 200 & \\
\hline & & $\begin{array}{l}\text { Les testicules } \\
\text { retirez dans le } \\
\text { corps 126 }\end{array}$ \\
\hline & & Cascar della matrice 201 & \\
\hline & & Pregnezza delle caualle 204 & \\
\hline & & Parto 204 & \\
\hline & & Secondine 206 & \\
\hline & $\begin{array}{l}\text { Sconciamento delle caualle } \\
206\end{array}$ & \\
\hline & $\begin{array}{l}\text { Far disgrauidare le caualle } \\
\text { pregne 207 }\end{array}$ & \\
\hline
\end{tabular}

Tabla 6. Patologías del sistema reproductor

\subsection{Patologías de las articulaciones y del esqueleto}

En las patologías de las articulaciones y del esqueleto, se hallan correspondencias etimológicas entre el portugués y el francés con respecto a las enfermedades de esforço ou rendimento das pas, cavalo aberto y esforço ou rendimento dos rins. Hay correspondencias etimológicas entre el portugués, el castellano y el italiano con respecto a la patología deslocações. En la patología curvas, de significado no tumoral, hay correspondencias entre el portugués y el italiano.

\begin{tabular}{|c|c|c|c|}
\hline REGO & PARACUELLOS & RUINI & SOLLEYSEL \\
\hline $\begin{array}{l}\text { Esforço ou rendimento } \\
\text { das pas } 279\end{array}$ & & $\begin{array}{l}\text { Dislocatione della spalla } \\
225 ? ? ?\end{array}$ & $\begin{array}{l}\text { Effort d'epaule, de } \\
\text { l'écart, ou du } \\
\text { cheval entr'ouvert } \\
132\end{array}$ \\
\hline \multirow[t]{8}{*}{ Cavalo aberto 282} & & & $\begin{array}{l}\text { du cheval } \\
\text { entr'ouvert } 138\end{array}$ \\
\hline & & $\begin{array}{l}\text { Male articolare in vniuersale } \\
\text { (sciatica, podagra) } 209\end{array}$ & \\
\hline & & Sciatica 213 & \\
\hline & & $\begin{array}{l}\text { Doglia della giuntura della } \\
\text { spalla } 214\end{array}$ & \\
\hline & & $\begin{array}{l}\text { Doglia delle giunture delle } \\
\text { ginocchia, \& delle pastore } 215\end{array}$ & \\
\hline & & Podagra 215 & \\
\hline & & Doglia della spalla 216 & \\
\hline & & Doglia della grassella 221 & \\
\hline \multirow[t]{4}{*}{ Deslocações 296} & dislocaciones & $\begin{array}{l}\text { Della Dislocatione in vni- } \\
\text { uersale } 223 \text { (lussatione } \\
\text { =exarthrema; sublussatione) }\end{array}$ & \\
\hline & & Dislocatione della spalla 225 & \\
\hline & & $\begin{array}{l}\text { Dislocatione dell'osso della } \\
\text { coscia } 227\end{array}$ & \\
\hline & & $\begin{array}{l}\text { Dislocationi delle gambe } \\
228\end{array}$ & \\
\hline
\end{tabular}




\begin{tabular}{|c|c|c|c|}
\hline & & Storta delle gambe 228 & \\
\hline \multirow[t]{10}{*}{$\begin{array}{l}\text { mãos pizadas, \& incha- } \\
\text { das p. } 286\end{array}$} & & & $\begin{array}{l}\text { Coups de pieds, } \\
\text { jambes enflées ou } \\
\text { gorgées } 151 \\
\text { Boulets enflez, ou } \\
\text { gorgez } 183\end{array}$ \\
\hline & & & $\begin{array}{l}\text { Jambes travaillées, } \\
\text { foulées, ou usées } \\
149\end{array}$ \\
\hline & Fracturas & $\begin{array}{l}\text { Della Rottura dell'ossa in } \\
\text { vniuersale } 229\end{array}$ & $\begin{array}{l}\text { Des jambes cas- } \\
\text { sées, \& des os } \\
\text { rompus des che- } \\
\text { vaux } 148\end{array}$ \\
\hline & & $\begin{array}{l}\text { Rottura dell' ossa delle } \\
\text { gambe } 229\end{array}$ & \\
\hline & & Ferite delle gambe 231 & \\
\hline & & & $\begin{array}{l}\text { Vieille enflure de } \\
\text { jambes, par une } \\
\text { nerferure qui } \\
\text { auroit esté mal } \\
\text { guerie } 156\end{array}$ \\
\hline & & $\begin{array}{l}\text { Fessura de i nerui, \& tendini } \\
\text { delle gambe } 233\end{array}$ & \\
\hline & & $\begin{array}{l}\text { Nerui tagliato alquanto per } \\
\text { lo trauerso } 234\end{array}$ & \\
\hline & & Neruo tronco 233 & \\
\hline & & Punture de i nerui 236 & \\
\hline \multirow[t]{3}{*}{$\begin{array}{l}\text { deslocação, \& esfria- } \\
\text { mento da junta } 296\end{array}$} & & Cauallo attinto 237 & $\begin{array}{l}\text { Entorses, \& dislo- } \\
\text { cations du boulet } \\
176\end{array}$ \\
\hline & & & Atteinte 186 \\
\hline & & $\begin{array}{l}\text { Tendini interiori delle brac- } \\
\text { cia del cauallo attinti } 238\end{array}$ & \\
\hline \multirow[t]{5}{*}{$\begin{array}{l}\text { Relaxação e desloca- } \\
\text { mento dos nervos } 414, \\
292 ? ?\end{array}$} & & Storte de i nerui 239 & Nerfs ferus 181 \\
\hline & & $\begin{array}{l}\text { Nerui, \& tendini duri, \& } \\
\text { ritirati } 240\end{array}$ & \\
\hline & & Ferite de i ligamenti 240 & \\
\hline & & Attritione de i ligamenti 241 & \\
\hline & & Storte de i ligamenti 241 & \\
\hline $\begin{array}{l}\text { curvas, \& sobre-curvas, } \\
\& \text { curvaças } 420\end{array}$ & & $\begin{array}{l}\text { Curuatione, \& estensione de } \\
\text { i nerui, e muscoli delle mani } \\
\text { del cauallo, per le quali sono } \\
\text { chiamati corbi, \& affaticati } \\
241 \\
\text { Curua, ò corba; curua, corba } \\
\text { alla rouerscia } 241\end{array}$ & \\
\hline $\begin{array}{l}\text { Do esforço ou rendimen- } \\
\text { to dos rins } 408\end{array}$ & & & $\begin{array}{l}\text { Effort de reins ou } \\
\text { chûtes } 448\end{array}$ \\
\hline
\end{tabular}




\begin{tabular}{|l|l|l|l|}
\hline $\begin{array}{l}\text { extensão e relaxamento } \\
\text { do nervo do jarrete 414 }\end{array}$ & & $\begin{array}{l}\text { Effort de jarret } \\
466\end{array}$ \\
& & & $\begin{array}{l}\text { Gros nerf du jarret } \\
\text { étendu \& forcé, \& } \\
\text { pour nerf feru 469 }\end{array}$ \\
\hline & & $\begin{array}{l}\text { Crampe ou gram- } \\
\text { pe 474 } \\
\text { [cãibra] }\end{array}$ \\
\hline
\end{tabular}

Tabla 7. Patologías de las articulaciones y esqueleto

\subsection{Patologías de los miembros de locomoción como grietas o semejantes}

Las patologías que afectan a las patas de los caballos y que no resultan en tumores son tratadas en seguida. Los términos portugueses greta, raças, alcançadura, arestins, galapago encuentran correspondencia etimológica con el castellano. Entre el portugués, el italiano y el francés hay correspondencias etimológicas relativas a porrilla y a cascos encastelados. Existen correspondencias del mismo tipo entre los cuatro manuales con respecto a quarto, encravadura, gavarro y figo. Entre el portugués, el castellano y el italiano, la correspondencia etimológica está en la patología formigueyro.

\begin{tabular}{|c|c|c|c|}
\hline REGO & PARACUELLOS & RUINI & SOLLEYSEL \\
\hline- & & Intrafregatura 264 & \\
\hline- & & $\begin{array}{l}\text { Infiammatione delle pastore } \\
264\end{array}$ & \\
\hline \multirow[t]{2}{*}{$\begin{array}{l}\text { mataduras, chagas; \& } \\
\text { feridas } 326\end{array}$} & & & Playes 275 \\
\hline & & $\begin{array}{l}\text { Creppature nelle gambe in } \\
\text { generale } 265 \text { : } \\
5 \text { tipos: } \\
\text { rappe } \\
\text { melandres } 265 \\
\text { Creppaccie, serpentine } 267 \\
\text { Crepaccie trauerse } 269\end{array}$ & \\
\hline \multirow[t]{2}{*}{$\begin{array}{l}\text { Gretas } 290 \text { [explicita } \\
\text { malandres en italiano] } \\
296 \mathrm{cf}\end{array}$} & grietas & Melandres 265, 267 & $\begin{array}{l}\text { Malandres \& } \\
\text { solandres } 163\end{array}$ \\
\hline & & & Solandres 163 \\
\hline- & & Rappe 265 & \\
\hline - & & $\begin{array}{l}\text { Creppaccie, \& serpentine } \\
267\end{array}$ & Crevasses? 495 \\
\hline- & & Crepaccie trauerse 269 & \\
\hline- & & Reste 269 & $\begin{array}{l}\text { Queues de rat, ou } \\
\text { arrestes } 492 \text { ?? } \\
\text { será reste de rui- } \\
\text { ni? }\end{array}$ \\
\hline \multirow[t]{2}{*}{ Arestins 301} & arestin & Rizzoli 271 & Peignes 268 \\
\hline & & ?? Crepaccie trauerse 269 & $\begin{array}{l}\text { Mulles traversieres } \\
493\end{array}$ \\
\hline Porrilha??? & & $\begin{array}{l}\text { Male de i fettoni simile à } \\
\text { porri 284??? }\end{array}$ & Poireaux 493 \\
\hline
\end{tabular}




\begin{tabular}{|c|c|c|c|}
\hline & & & $\begin{array}{l}\text { Mauvaises Eaux } \\
496\end{array}$ \\
\hline$?$ & & $?$ & Crapaudines 509 \\
\hline$?$ & & $?$ & $\begin{array}{l}\text { Étonnement du } \\
\text { sabot } 510\end{array}$ \\
\hline$?$ & & $\begin{array}{l}\text { Riprensione, ouero infusione } \\
272\end{array}$ & \\
\hline \multirow[t]{2}{*}{$\begin{array}{l}\text { manqueira por defeito, } \\
\text { ou falta de cascos. Por } \\
\text { "muyto molles, humi- } \\
\text { dos, a que chamaõ [Cas- } \\
\text { qui molli,], outros por } \\
\text { muyto asperos, \& que- } \\
\text { bradiços, a que chamão } \\
\text { [Casqui vidroso,]" p. } \\
320\end{array}$} & & $\begin{array}{l}\text { Mali dell'vgne; \& dell'vgne } \\
\text { troppo secche, \& vetriole } \\
274 \\
\text { Vgne troppo tenere ; \& molli } \\
276\end{array}$ & \\
\hline & & $\begin{array}{l}\text { Fessure dell'vgne: Quarto, } \\
\text { Setola, Rotture dell'vgne, } \\
\text { Mal dell'asino }\end{array}$ & \\
\hline Quarto 311 & quarto & Quarto 277 & $\begin{array}{l}\text { Quartes, ou pieds } \\
\text { de bouefs } 509 \\
\text { Seymes, ou pieds } \\
\text { fendus, nommez } \\
\text { pieds de boeuf } 219 \\
\end{array}$ \\
\hline \multirow[t]{2}{*}{ Raças 311} & raza & & \\
\hline & & Setola 281 & \\
\hline- & & Rotture dell'vgne 282 & \\
\hline$?$ & & Mal dell'asino 283 & \\
\hline$?$ & & Creppature de i fettoni 283 & \\
\hline$?$ & & $\begin{array}{l}\text { Male de i fettoni simile à } \\
\text { porri } 284\end{array}$ & \\
\hline- & & $\begin{array}{l}\text { Separatione dell'vgna del } \\
\text { viuo del piede } 285\end{array}$ & \\
\hline Encravadura 315 & enclavadura & Inchiodatura 285 & Encloüeure, 237 \\
\hline Encravadura 315 & $\begin{array}{l}\text { Puntura de clavo o } \\
\text { de otra cosa }\end{array}$ & Isprocatura 289 & $\begin{array}{l}\text { clous de ruë, ou } \\
\text { chicots } 240\end{array}$ \\
\hline Alcançadura?? & $\begin{array}{l}\text { Cazapatazo?? } \\
\text { alcanzaduras }\end{array}$ & Sopraposta 290 & \\
\hline \multirow[t]{2}{*}{$\begin{array}{l}\text { Pancada do casco ou } \\
\text { ferradura assentada }\end{array}$} & $\begin{array}{l}\text { Assentadura de } \\
\text { herradura }\end{array}$ & & \\
\hline & & Contusione de i piedi 291 & \\
\hline Formigueyro 325????? & Hormiguilla??? & Formica, ò carvolo 294 & \\
\hline $\begin{array}{l}\text { Gavarro } 305 \\
3 \text { generos espanhóis só } \\
\text { um } \\
\text { Gavarro a q os franceses } \\
\text { chamam encornado } \\
\text { Gavarro nervudo } \\
\text { Gavarro simplez }\end{array}$ & $\begin{array}{l}\text { Gavarro } 88 \\
1 \text { género }\end{array}$ & $\begin{array}{l}\text { Chiouardo, ò clauardo, ò } \\
\text { gauaro } 294\end{array}$ & $\begin{array}{l}\text { Javar } 187 \\
3 \text { géneros: } \\
\text { Simples } \\
\text { Nerveux } \\
\text { Encornez } 198 \\
\text { (javar encorné ou } \\
\text { atteinte encornée } \\
\text { 198) }\end{array}$ \\
\hline- & & Pinzanese 295 & \\
\hline
\end{tabular}




\begin{tabular}{|c|c|c|c|}
\hline & $\begin{array}{l}\text { Enrejadura } 94 \\
\text { ferida feita com as } \\
\text { rejas }\end{array}$ & & Embarrures 467 \\
\hline Galapago 308 & Galapago & & \\
\hline \multirow{4}{*}{$\begin{array}{l}\text { podridão, ou figo das } \\
\text { ranilhas, \& formigueyro } \\
324\end{array}$} & Higos & Fico 296 & $\begin{array}{l}\text { Fics ou crapeaux } \\
230\end{array}$ \\
\hline & & $?$ & Teignes 267 \\
\hline & & & $\begin{array}{l}\text { Des maux de la } \\
\text { fourchette qui sont } \\
\text { des boüillons de } \\
\text { chair, ou des } \\
\text { cerises que les } \\
\text { ignorans prennent } \\
\text { pour des fics } 270\end{array}$ \\
\hline & & $\begin{array}{l}\text { Malla compositione } \\
\text { dell'vgne, \& de i piedi del } \\
\text { cauallo } 296\end{array}$ & \\
\hline$?$ & & $i$ & $\begin{array}{l}\text { Méchands pieds } \\
213\end{array}$ \\
\hline$?$ & & $i$ & Pieds solbatus 216 \\
\hline \multirow[t]{3}{*}{$\begin{array}{l}\text { cascos enchapinados, ou } \\
\text { encastelados } 309\end{array}$} & & $\begin{array}{l}\text { Incastellatura de' piedi } \\
\text { dinanzi }\end{array}$ & $\begin{array}{l}\text { Pieds encastelez } \\
223\end{array}$ \\
\hline & & & Bleymes 265 \\
\hline & & & $\begin{array}{l}\text { Pied privé de } \\
\text { nourriture } 273\end{array}$ \\
\hline$?$ & & Cerchi delli piedi dinanzi 298 & \\
\hline \multirow[t]{4}{*}{ Cavalos topinhos 423} & & Piedi codogni 299 & \\
\hline & & $\begin{array}{l}\text { Piedi dinanzi depressi, \& } \\
\text { piani } 300\end{array}$ & \\
\hline & & Piedi torti 300 & \\
\hline & & Piedi rampini, ò mancini 300 & \\
\hline
\end{tabular}

Tabla 8. Patologías de los miembros de locomoción como grietas o semejantes

\subsection{Patologías de los miembros de locomoción como tumores}

En las patologías de las patas que derivan en tumores, hay correspondencias etimológicas entre los cuatro manuales en la enfermedad de curvas, sobrecurvas y curvaças. Entre el portugués y el castellano, se corresponden etimológicamente las designaciones de agrião, alifafes y eslabão. Entre el portugués, el francés y el castellano se corresponden los términos referentes a lupas. Entre el portugués, el italiano y el francés, hay correspondencias referidas a esparavões.

\begin{tabular}{|l|l|l|l|}
\hline REGO & PARACUELLOS & RUINI & SOLLEYSEL \\
\hline Agrião 416 & & Spauento 245 & \\
\hline Sobrecana 291 & Agriones 151 & Cappelletto 245 & Capelets 475 \\
\hline Ovas 294 & & $i$ & $\begin{array}{l}\text { Sur-os fusées, \& } \\
\text { osselets 164 }\end{array}$ \\
\hline
\end{tabular}




\begin{tabular}{|c|c|c|c|}
\hline Alifafes 417 & Alifafes 165 & $\begin{array}{l}\text { Vescicone del garettone, ò } \\
\text { ginocchio } 246\end{array}$ & $\begin{array}{l}\text { Vessigons 169, } \\
467\end{array}$ \\
\hline$?$ & & Iarda, ò zarda 253 & $\begin{array}{l}\text { Jardon ou jardé } \\
480\end{array}$ \\
\hline \multirow[t]{2}{*}{ ????? } & & Galle 254 & \\
\hline & & & Varisses 483 \\
\hline $\begin{array}{l}\text { Esparavões } 421 \\
\text { Seco ou intrinsecos } 421 \text { - } \\
422 \\
\text { Boyuno } 422\end{array}$ & & $\begin{array}{l}\text { Sparagagno, ò sparauano } \\
257\end{array}$ & $\begin{array}{l}\text { Esparvins } 467,481 \\
\text { Esparvin sec } 481 \\
\text { Esparvin de Boeuf } \\
482\end{array}$ \\
\hline \multirow[t]{4}{*}{$\begin{array}{l}\text { sobre-mãos, ou formas, } \\
\& \text { cravos p. } 303 \\
\text { sobre-mãos os Fran- } \\
\text { cezes lhe chamam for- } \\
\text { mas } 303\end{array}$} & \multirow[t]{2}{*}{$\begin{array}{l}\text { Esparavanes, y } \\
\text { sobrehuessos, y } \\
\text { cabeza, y sobre- } \\
\text { mano, y pie, y } \\
\text { clavo passado } 137\end{array}$} & & \\
\hline & & & Formes 209 \\
\hline & & Sopra osso 258 & $\begin{array}{l}\text { Sur-os fusées, \& } \\
\text { osselets } 164\end{array}$ \\
\hline & & $\begin{array}{l}\text { Chiapponi } 264 \\
\text { Formella } 261\end{array}$ & \\
\hline Sobre-mão & & Mazzuola 261 & \\
\hline \multicolumn{4}{|l|}{$?$} \\
\hline \multicolumn{4}{|l|}{$?$} \\
\hline \multirow[t]{2}{*}{$\begin{array}{l}\text { curvas, \& sobre-curvas, } \\
\& \text { curvaças } 420\end{array}$} & corbaza & Curba 258 & Courbes 467,484 \\
\hline & & & $\begin{array}{l}\text { Des tous les maux } \\
\text { des jambes de } \\
\text { derriere, du jarret } \\
\text { en bas } 491\end{array}$ \\
\hline Eslabão 290 & Eslabones 141 & & \\
\hline Lupas 289 & Lupias 145 & & Louppes 169 \\
\hline agrião 416 & Agriones 151 & & \\
\hline
\end{tabular}

\subsection{Patologías cardíacas}

Con respecto a las patologías cardíacas, se hallan correspondencias etimológicas entre el portugués, el francés y el italiano en la patología antecor.

\begin{tabular}{|l|l|l|l|}
\hline REGO & PARACUELLOS & RUINI & SOLLEYSEL \\
\hline & & Mali del cuore 126: tipos: & \\
\hline $\begin{array}{l}\text { Latejar do coração 276- } \\
277\end{array}$ & Dolore del cuore 126 & \\
\hline & $\begin{array}{l}\text { Palpitatione, 126 batticuore } \\
130\end{array}$ & $\begin{array}{l}\text { Battement du } \\
\text { coeur ou palpita- } \\
\text { tion 367 }\end{array}$ \\
\hline $\begin{array}{l}\text { Ante cor 'tumor no } \\
\text { peito, pescoço' 273 }\end{array}$ & $\begin{array}{l}\text { Lobarro 98 no } \\
\text { peito, pescoço }\end{array}$ & Sincopa 126 & \\
\hline
\end{tabular}

Tabla 10. Patologías cardíacas 


\subsection{Patologías oftálmicas}

Las patologías oculares demuestran paralelismo etimológico entre Rego, Ruini y Solleysel relativamente a cavallo lunatico. Entre Rego y Solleysel hay paralelismo en fluxão dos olhos.

\begin{tabular}{|c|c|c|c|}
\hline REGO & PARACUELLOS & RUINI & SOLLEYSEL \\
\hline \multicolumn{4}{|l|}{ Nevoa dos olhos 252} \\
\hline & $\begin{array}{l}\text { Nubecillas ou } \\
\text { paños } 59,63 ? ? \mathrm{Ou} \\
\text { albugem? }\end{array}$ & cataratta & \\
\hline Cavallo lunatico 252 & & Cauallo lunatico 80 & $\begin{array}{l}\text { Cheval lunatique } \\
84\end{array}$ \\
\hline- & & $\begin{array}{l}\text { Cauallo ombroso (por vista } \\
\text { má) } 81\end{array}$ & \\
\hline- & & $\begin{array}{l}\text { Albugine ò panno } \\
\text { dell'occhio } 83\end{array}$ & \\
\hline- & & $\begin{array}{l}\text { Tumori, ò pustule } \\
\text { dell'occhio } 85\end{array}$ & \\
\hline- & & Acino dell'occhio 86 & \\
\hline- & & Vlceri de gli occhi 87 & \\
\hline \multirow[t]{2}{*}{-} & & Macchie rosse de gli occhi 87 & \\
\hline & & Vnghiella de gli occhi 88 & \\
\hline \multirow[t]{2}{*}{ Fluxão dos olhos 249} & & Lagrime (griego epifora) 89 & $\begin{array}{l}\text { Maux des yeux } \\
\text { fluxion } 73\end{array}$ \\
\hline & & Oftalmia ò lippitudine 91 & \\
\hline \multirow[t]{4}{*}{$\begin{array}{l}\text { Pancada ou golpe sobre } \\
\text { o olho } 251\end{array}$} & & Ferite ne gli occhi 94 & $\begin{array}{l}\text { Coups, heurts \& } \\
\text { morsures sur l'oeil } \\
80\end{array}$ \\
\hline & & Percosse ne gli occhi 97 & \\
\hline & & Grattature dell'occhio 98 & \\
\hline & & Tritiace 99 & \\
\hline
\end{tabular}

Tabla 11. Patologías oftálmicas

\subsection{Patologías respiratorias}

Por último, en las patologías respiratorias hay paralelismo etimológico entre el portugués, el italiano y el francés con respecto a tosse.

\begin{tabular}{|l|l|l|l|}
\hline REGO & PARACUELLOS & RUINI & SOLLEYSEL \\
\hline $\begin{array}{l}\text { Polmoeira Dar aos foles } \\
341\end{array}$ & Nerfago 106 & $\begin{array}{l}\text { Difficultà del respirare 131: } \\
\text { 3 tipos: }\end{array}$ & Pousse 314 \\
\hline & & $\begin{array}{l}\text { Difficultà di rifiatare ò fiato } \\
\text { grosso 131 }\end{array}$ & \\
\hline & Asma ò sospiro 131 & \\
\hline & & Orthopnea 131 & \\
\hline & & Peripleumonia 139 & \\
\hline Tosse 344 & Bolso 140-141 & \\
\hline & $\begin{array}{l}\text { Tosse 2 tipos secca; } \\
\text { humida143 }\end{array}$ & Toux 331 \\
\hline
\end{tabular}




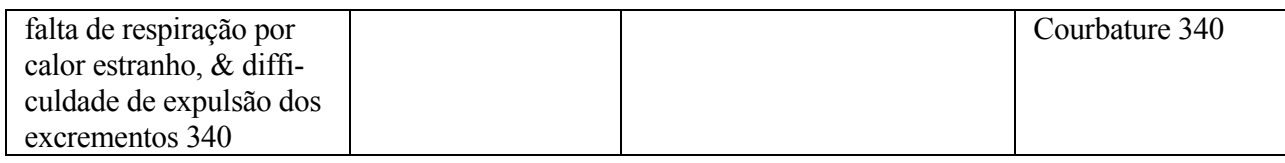

Tabla 12. Patologías respiratorias

\section{CONCLUSIONES}

Debido a la discrepancia organizativa y de abordaje de cada una de las obras analizadas, existen muchas patologías que son tratadas de modos distintos por cada uno de los autores. Además, Paracuellos es el autor menos completo, al paso que Ruini enfoca un gran número de patologías. Por esos motivos, los resultados obtenidos no pueden ser tomados como indicadores absolutos de la terminología referente de las patologías hipiátricas del siglo XVII en portugués, en castellano, en francés y en italiano, una vez que no todas se encuentran descritas en todos los tratados analizados. Así, los resultados solamente son válidos para la comparación terminológica entre los autores nombrados. Con base en los datos analizados, no es posible establecer una influencia más fuerte de un determinado tratado sobre la obra de Rego. La terminología de Rego muestra transversalidad con el castellano, el francés y el italiano, como es de esperar en un dominio cuya permeabilidad cultural ha sido muy fuerte desde la Edad Media.

\section{REFERENCIAS BIBLIOGRÁFICAS}

Mestre Giraldo ([1318] 1909): Livro d'alveitaria, en Gabriel Pereira, ed., Revista Lusitana XII: 1-60.

PARACUellos, Miguel de (1658): Libro de albeyteria, Zaragoça, por Iuan de Ybar a costa de Tomas Cabeças, mercader de libros.

REGO, António Pereira (1693): Instruçam da Cavallaria de Brida: tratado unico, dedicada ao invicto martyr S. Jorge, Coimbra, na Off. de Joam Antunes.

RODRIGUES, Alexandra Soares (2012a): «El léxico de la albeitería en el Vocabulario portuguez e latino de Bluteau (1712-1728)», en Graça Rio-Torto, org., Léxico de la Ciencia: tradición y modernidad, Múnich, Lincom Europa, pp. 163-178.

RODRIGUES, Alexandra Soares (2012b): «Los términos de enfermedades en el Livro d'Alveitaria de Mestre Giraldo», en Julia Pinilla Martínez, Virginia González García y Cecilio Garriga Escribano, eds., Quaderns de Filologia. Estudis Lingüístics, XVII: Lengua y ciencia. Recepción del discurso científico, pp. 243-256.

RODRIGUES, Alexandra Soares (2013): «Los nombres de enfermedades de los caballos en el portugués del siglo XVII: influencias», en Carsten Sinner, ed., Comunicación y transmisión del saber entre lenguas y culturas, Études linguistiques/Linguistische Studien Band 10, Múnich, Peniope, pp. 83-98.

RODRIGUES, Alexandra Soares (2016): «Adragunchos voadjos, anafafes y exaaguases: términos no construidos en Mestre Giraldo y su destino en la historia del portugués», en Cecilio Garriga Escribano y José Ignacio Pérez Pascual, eds., Lengua de la ciencia e historiografia, A Coruña, Universidade da Coruña, pp. 283-296. 
RoDRIGUES, Alexandra Soares (2017a): «Correlación entre los nombres de enfermedades en el Livro de Alveitaria de Mestre Giraldo y en sus fuentes (el Liber de Medicina Equorum de Giordano Ruffo y el Mulomedicina de Teodorico Borgognoni)», presentado en la VI Reunión Internacional de la Red Temática Lengua y Ciencia, Madrid, 22-24 de febrero de 2017.

RODRIGUES, Alexandra Soares (2017b): «Sostra vs. danadura do espiñaço; eslomedramento vs. door da anca: léxico romance en Galicia y en Portugal en los tratados medievales de albeitería», presentado en el VII Coloquio Internacional sobre la Historia de los Lenguajes Iberorrománicos de Especialidad (CIHLIE), Alcalá de Henares, 19-20 de octubre de 2017.

Rodrigues, Alexandra Soares y Luísa Inês SoARes SÁ MorAis (2015): «Los términos de albeitería en Mestre Giraldo: ¿continuidad a lo largo de los siglos?» en Jenny Brumme y Carmen López Ferrero, eds., La ciencia como diálogo entre teorías, textos y lenguas, Berlín, Frank \& Timme, pp. 13-30.

RUINI, Carlo (1618): Anatomia del cavallo, infermita et suoi remedii, Venecia, Fiorauante Prati.

SOLLEYSEL, Jacques de (1654): Le Parfait mareschal, París, Gerv.Clousier. 